\title{
MINIMUM DETERMINANT OF ASYMMETRIC QUADRATIG FORMS
}

\author{
R. T. WORLEY
}

(Received 1 September 1965, revised 30 June 1966)

1

Let $f=f\left(x_{1}, x_{2}, \cdots, x_{n}\right)$ be an indefinite $n$-ary quadratic form of signature $s$ and let $m_{+}(f), m_{-}(f)$ denote the infimum of the non-negative values taken by $f$ and $-f$ respectively for integral $\left(x_{1}, x_{2}, \cdots, x_{n}\right) \neq$ $(0,0, \cdots, 0)$. Furthermore let $f$ satisfy the condition $m_{+}(f) \neq 0$ and let

$$
A(f)=\frac{m_{-}(f)}{m_{+}(f)} \geqq k
$$

for some integer $k$. Then Segré [3] has shown that, for $n=2, f$ must have determinant $\operatorname{det}(f)$ satisfying

$$
|\operatorname{det}(f)| \geqq\left(m_{+}(f)\right)^{2}\left(k^{2}+4 k\right) / 4 \text {, }
$$

with equality if and only if $f$ is equivalent under an integral unimodular transformation (denoted $\sim$ ) to a multiple of the form $f_{1}(x, y)=$ $x^{2}-k x y-k y^{2}$, while Oppenheim [2] has shown that, for $n \geqq 3$,

is of the order of $k^{2 n-2}$.

$$
\frac{|\operatorname{det}(f)|}{\left(m_{+}(f)\right)^{n}}
$$

In this paper the results of Tornheim [4] are used to extend Segre's result above, and this extension is used to find a bound, of the order of $k^{2 n-2}$, such that there are only finitely many non-equivalent indefinite ternary quadratic forms $f$ which attain the value $m_{+}(t)=1$ and which have $d(f)=$ $|\operatorname{det}(f)|$ less than this bound. The following are the theorems proved.

THEOREM 1. For integral $k \geqq 2$ there exists a positive constant $c(k)$ such that whenever an indefinite binary quadratic form $q=q(x, y)$ satisfies

$$
0<(1-c) m_{+}(q) \leqq m_{-}(q) / k
$$

for some $c$ with $0 \leqq c<c(k)$ it may be concluded that either

(i) $q \sim m_{+}(q)\left(x^{2}-k x y-k y^{2}\right)$ and $A(q)=k$, or

(ii) $d(q) \geqq\left[m_{+}(q)\right]^{2}(1-c)^{2}\left(k^{2}+6 k+1\right) / 4$. 
Theorem 2. Let $k \geqq 2$ be integral and define

$$
\begin{aligned}
K & =k^{2}+6 k+1, \\
t(S) & =K^{2}(1+4 / S) / 64, \\
d_{1} & =\left(K^{2}+12 K\right) / 64, \\
d_{2} & =\max \left(\min \left\{t(S), 9(S+\sqrt{ } 5)^{2} / 64\right\}\right),
\end{aligned}
$$

where the maximum is taken over all positive integers $S$, and let $S^{*}$ denote the $S$ at which the maximum is attained. For positive integers $r$ and $s$ let $q(r, s ; y, z)$ denote the indefinite binary quadratic form

$$
y^{2}-\frac{s(r+2)}{r s+r+s} y z-\frac{r+2}{r s+r+s} z^{2},
$$

and for integral $l, 0 \leqq l<s$, let $f(r, s, l ; x, y, z)$ denote the indefinite ternary quadratic form

$$
\left(x+\frac{k}{2} y+\frac{l}{s} z\right)^{2}-\frac{1}{4}\left(k^{2}+4 k\right) q(r, s ; y, z) .
$$

Let $f=f(x, y, z)$ be an indefinite ternary quadratic form of signature 1 with $d(f)=d$ such that

(i) $m_{+}(f)=1$ and this value is attained by $f$, and

(ii) $m_{-}(f) \geqq k$.

Then either

(a) $d \geqq \min \left(d_{1}, d_{2}\right)$, or

(b) $m_{-}(f)=k$ and $f \sim f(r, s, l ; x, y, z)$ for some $r$ and $s$ such that $r \leqq s \leqq S^{*}$.

Theorem 1 may be used to obtain information about indefinite binary quadratic forms $q$ that have asymmetry $A(q)$ slightly below an integer $k$. It is clear from the statement of the theorem that if $q$ is an indefinite binary quadratic form with

$$
k(1-c(k))<A(q)<k
$$

then setting $c=1-A(q) / k$ yields that

$$
d(q) \geqq\left[m_{-}(q)\right]^{2}\left(k^{2}+6 k+1\right) / 4 k^{2} .
$$

In addition, the following corollary to theorem 1 should be noted.

COROLlARY TO THEOREM 1 . If $k \geqq 2$ is integral and if $q=q(x, y)$ is an indefinite binary quadratic form with $m_{+}(q)=1$ and $m_{-}(q) \geqq k+l$ where $l>0$, then

$$
d(q) \geqq \frac{1}{4}\left(k^{2}+6 k+1\right)+l .
$$

It should be noted that the condition that $f$ should attain the value $m_{+}(f)=1$ can be removed to make theorem 2 apply to all forms $f$ with 
$m_{+}(f)=1$ and $m_{-}(f) \geqq k$. This can be done modifying the argument given in this paper to allow for the fact that $m_{+}(f)$ may not be attained.

It should also be noticed that not all forms $f(r, s, l ; x, y, z)$ have $m_{+}(f)=1$ and $m_{-}(f)=k$. In fact it appears to be the exception rather than the rule that a form shall satisfy this condition. Calculations performed on the C.S.I.R.O.'s C.D.C. " 3200 " computer in Adelaide have shown that for $k=7,10,11$ and 12 not one of the forms has $m_{+}(f)=1, m_{-}(f)=k$ and $d(f)<\min \left(d_{1}, d_{2}\right)$, while for $k=2,3,4,5,6,8$ and 9 the forms listed in table 1 were found to be the only ones satisfying these constraints (note: for simplicity in the table the transformation $x \rightarrow x-[k / 2] y$ has been performed, where $[k / 2]$ denotes the integer part of $k / 2$ ).

For comparison with the determinants of the forms listed in table 1 , $\min \left(d_{1}, d_{2}\right)$ is listed in table 2 .

TABLE 1

\begin{tabular}{lll}
\hline$k$ & form & $r, s, l, d(f)$ \\
\hline 2 & $\left(x+\frac{1}{2} z\right)^{2}-3\left(y^{2}-y z-\frac{1}{4} z^{2}\right)$ & $4,4,2,4 \frac{1}{2}$ \\
2 & $\left(x+\frac{1}{2} z\right)^{2}-3\left(y^{2}-y z-\frac{1}{2} z^{2}\right)$ & $2,2,1,6 \frac{3}{4}$ \\
2 & $x^{2}-3\left(y^{2}-\frac{4}{3} y z-\frac{1}{3} z^{2}\right)$ & $1,4,0,7$ \\
3 & $\left(x+\frac{1}{2} y\right)^{2}-\frac{21}{4}\left(y^{2}-\frac{8}{7} y z-\frac{2}{7} z^{2}\right)$ & $2,4,0,16 \frac{7}{8}$ \\
4 & $x^{2}-8\left(y^{2}-y z-\frac{1}{8} z^{2}\right)$ & $8,8,0,24$ \\
5 & $\left(x+\frac{1}{2} y+\frac{1}{2} z\right)^{2}-\frac{45}{4}\left(y^{2}-\frac{6}{5} y z-\frac{1}{15} z^{2}\right)$ & $3,18,9,54$ \\
6 & $\left(x+\frac{1}{2} z\right)^{2}-15\left(y^{2}-y z-\frac{1}{20} z^{2}\right)$ & $20,20,10,67 \frac{1}{2}$ \\
6 & $\left(x+\frac{1}{2} z\right)^{2}-15\left(y^{2}-y z-\frac{1}{6} z^{2}\right)$ & $6,6,3,93 \frac{3}{8}$ \\
6 & $x^{2}-15\left(y^{2}-\frac{6}{5} y z-\frac{1}{15} z^{2}\right)$ & $3,18,0,96$ \\
8 & $x^{2}-24\left(y^{2}-y z-\frac{1}{9} z^{2}\right)$ & $9,9,0,208$ \\
9 & $\left(x+\frac{1}{2} y+\frac{1}{2} z\right)^{2}-\frac{117}{4}\left(y^{2}-\frac{14}{13} y z-\frac{1}{39} z^{2}\right)$ & $9,42,21,270$ \\
\hline
\end{tabular}

TABLE 2

\begin{tabular}{ccccccccc}
\hline$k$ & 2 & 3 & 4 & 5 & 6 & 7 & 8 & 9 \\
\hline $\min \left(d_{1}, d_{2}\right)$ & $7.5 \ldots$ & 17.5 & $33.7 \ldots$ & $59.3 \ldots$ & $96.7 \ldots$ & $149.3 \ldots$ & $220.5 \ldots$ & $314.1 \ldots$ \\
\hline
\end{tabular}

We shall now prove theorems 1 and 2 and the corollary to theorem 1.

Proof of THE COROLlary to Theorem 1 . Taking $c=0$ in theorem 1 we find that $d=d(q) \geqq\left(k^{2}+6 k+1\right) / 4$. Suppose that $d<\frac{1}{4}\left(k^{2}+6 k+1\right)+l$. Then as $m_{+}(q)=1$ we may write for arbitrarily small $\delta \geqq 0$ 


$$
q \sim \frac{1}{1-\delta}(x+\lambda y)^{2}-d(1-\delta) y^{2},
$$

and so by choosing $x$ such that

$$
\left(k^{2}+2 k+1\right) / 4 \leqq(x+\lambda)^{2} \leqq\left(k^{2}+4 k+4\right) / 4
$$

we obtain a value of $q$ which for sufficiently small $\delta$ lies in the open interval $(-k-l, 1)$. This contradicts either $m_{+}=1$ or $m_{-} \geqq k+l$.

Proof of THEOREM 1. The proof of this theorem depends upon the work of Tornheim [4]. We let

$$
Q(x, y)=q(x, y) / 2 \sqrt{d(q)},
$$

so that $Q$ is an indefinite binary quadratic form with discriminant $\Delta^{2}=1$.

We define

$$
\begin{aligned}
M & =m_{+}(q), \\
N & =m_{-}(q), \\
A & =\max (1 / M, k / N), \\
A_{1} & =\sqrt{k^{2}+4 k}, \\
A_{2} & =\left[k^{2}+k+(3 k-1) A_{1}\right] /(4 k-2), \\
c^{*}(k) & =1-A_{1} / A_{2}>0 .
\end{aligned}
$$

Then Tornheim has shown that either

(a) $A=A_{1}$ and $N=k M$, in which case $Q \sim M\left(x^{2}-k x y-k y^{2}\right)$, or

(b) $A \geqq \sqrt{k^{2}+6 k+1}$, or

(c) $M \geqq 1 / A_{1}$ and $N \leqq k / A_{2}$.

Consider firstly the third alternative. This implies that

and so

$$
N / k M \leqq A_{1} / A_{2}=1-c^{*}(k),
$$

$$
m_{-}(q) / k \leqq\left(1-c^{*}(k)\right) m_{+}(q) .
$$

Hence if we set $c(k)=c^{*}(k)$ we have, for $0 \leqq c<c(k)$, that

$$
m_{-}(q) / k<(1-c) m_{+}(q) \text {, }
$$

which contradicts the given. It remains to show that, with $c(k)=c^{*}(k)$, the conclusions (i) and (ii) of the theorem follow from the alternatives (a) and (b) above. Since (a) clearly implies (i) we need only show that (b) implies (ii).

From (b) we have that

and so

$$
\max (1 / M, k / N) \geqq \sqrt{k^{2}+6 k+1},
$$




$$
2 \sqrt{d(q)} \geqq \sqrt{k^{2}+6 k+1} \min \left(m_{+}(q), m_{-}(q) / k\right) .
$$

Using the given it follows that

as required.

$$
d(q) \geqq \frac{1}{4}\left[m_{+}(q)\right]^{2}(1-c)^{2}\left(k^{2}+6 k+1\right)
$$

In order to prove theorem 2 we need the following lemma on indefinite binary quadratic forms.

LEMMA 1. Let $q(x, y)$ be an indefinite binary quadratic form with $\Delta=1$ and let $\left[g_{i}\right]$ be the chain of positive integers associated with the chain of reduced forms equivalent to $q .{ }^{1}$

Suppose that the elements $g_{2 i}$ of the chain are bounded above by the integer $S$, and let

$$
C(S)=3 \sqrt{ } 5 / 40(S+1)^{2} .
$$

Let $M, N$ denote $m_{+}(q), m_{-}(q)$ respectively. Let $k \geqq 2$ be integral and let $c_{1}$ and $c_{2}$ be small positive numbers with $c_{1}<C(S)$ such that for each negative value $-n$ taken by $q$ either

$$
n / N \leqq 1+c_{1}
$$

or

$$
n / N \geqq\left(k^{2}+6 k+1\right) /\left(k^{2}+4 k\right)-c_{2} .
$$

\section{Then either}

(i) $1 / N \geqq 2$, or

(ii) $1 / N \geqq \sqrt{1+4 / S}\left[\left(k^{2}+6 k+1\right) /\left(k^{2}+4 k\right)-c_{2}\right]$, or

(iii) There exist integers $r$ and $s$, both at most $S$, such that for all integers $i$,

$$
g_{8 i+1}=1, \quad g_{4 i}=r, \quad g_{4++2}=s .
$$

Proof. If $g_{2 i+1} \geqq 2$ for any $i$, then $q$ takes the value $-n$ where

$$
1 / n=\left(g_{2 i+1}, g_{2 i+2}, g_{2 i+3}, \cdots\right)+\left(0, g_{2 i}, g_{2 i-1}, \cdots\right) .
$$

Hence $1 / n \geqq 2$, and as $n \geqq N$ it follows that $1 / N \geqq 2$.

We now suppose that $g_{2 i+1}=1$ for all $i$, and in addition that the chain is not of the form given in the third alternative. Clearly the proof of the lemma will be complete when we show that alternative (ii) must hold.

As the chain is not of the form in alternative (iii) there must exist an $i$ for which $g_{2 i} \neq g_{2 i+d}$. Let

1 It is assumed that the reader is familiar with the theory of reduced binary quadratic forms and the continued fractions associated with these forms. I use the notation of Dickson [1] with the exception that $\Delta^{2}$ and not $D^{2}$ is used to denote the discriminant. 
Let

$$
\begin{aligned}
& s=\max \left(g_{2 i}, g_{2 i+4}\right), \\
& t=\min \left(g_{2 i}, g_{2 i+4}\right), \quad \text { and } \\
& r=g_{2 i+2} .
\end{aligned}
$$

$$
\begin{aligned}
1 / n & =(1, r, 1, t, 1, \cdots)+(0, s, 1, \cdots) \\
& =(1, r, 1, \lambda)+(0, \mu)
\end{aligned}
$$

and

$$
\begin{aligned}
1 / n_{1} & =(1, r, 1, s, 1, \cdots)+(0, t, 1, \cdots) \\
& =(1, r, 1, \mu)+(0, \lambda),
\end{aligned}
$$

where the.. indicates the continuation of the chain in the expected manner, so that $-n$ and $-n_{1}$ are values taken by $q$. Consider the function

$$
\begin{aligned}
f(x) & =(0, r, x)-x \\
& =x /(1+r x)-x .
\end{aligned}
$$

Then the derivative $f^{\prime}(x)$ of $f(x)$ is given by

$$
f^{\prime}(x)=1 /(1+r x)^{2}-1,
$$

and so $f^{\prime}(x)<-\frac{3}{4}$ for $r \geqq 1$ and $x>1$. Now by the mean value theorem of calculus, as $f(x)$ is continuous and differentiable for $r \geqq 1$ and $x>1$, we have for $r \geqq 1$ and $1<x_{2}<x_{1}$ that

$$
f\left(x_{1}\right)-f\left(x_{2}\right)=\left(x_{1}-x_{2}\right) f^{\prime}(\alpha)
$$

for some $\alpha$ with $x_{2}<\alpha<x_{1}$. Substituting $x_{1}=(1, \lambda)$ and $x_{2}=(1, \mu)$ and simplifying, noting that $f^{\prime}(\alpha)<-\frac{3}{4}$, gives that

$$
1 / n-1 / n_{1}<-\frac{3}{4}(1 / \lambda-1 / \mu) \text {. }
$$

Now

$$
\begin{aligned}
\mu-\lambda & =(s, 1, \cdots)-(t, 1, \cdots) \\
& \geqq(s-t)+(0, \overline{1})-(0, \overline{1, S})^{2} \\
& >1+\frac{1}{2}-1 \\
& =\frac{1}{2}
\end{aligned}
$$

and $\mu \lambda<(S+1)^{2}$ as $\lambda$ and $\mu$ are each at most $S+1$. Hence

$$
1 / \lambda-1 / \mu>\frac{1}{2}(S+1)^{-2} \text {. }
$$

Using this in (1) yields that

$$
1 / n-1 / n_{1}<-\frac{3}{8}(S+1)^{-2} .
$$

2 A bar over portion of a continued fraction indicates that this portion is repeated indefinitely. 
Now as $1 / N \geqq 1 / n_{1}$ it follows that

$$
n / N-1>3 n / 8(S+1)^{2},
$$

from which, as $1 / n \leqq(1, \overline{1})+(0, \overline{1})=\sqrt{ } 5$, we can deduce that

$$
n / N>1+3 \sqrt{ } 5 / 40(S+1)^{2}=1+C(S)>1+c_{1} .
$$

Hence, using the given conditions, we must have

Now

$$
n / N \geqq\left(k^{2}+6 k+1\right) /\left(k^{2}+4 k\right)-c_{2} .
$$

$$
1 / n \geqq(\overline{1, S})+(0, \overline{S, 1})=\sqrt{1+4 / S,}
$$

and so we can conclude that

$$
1 / N \geqq \sqrt{1+4 / S}\left[\left(k^{2}+6 k+1\right) /\left(k^{2}+4 k\right)-c_{2}\right],
$$

which is alternative (ii) as required.

Proof of THEOREM 2. Let $f$ be an indefinite ternary quadratic form of signature 1 such that $m_{+}(f)=1, m_{-}(f) \geqq k$, and let $f$ attain the value 1 . By passing to a suitable equivalent form we may assume $f$ to be given in the form

$$
f=(x+\lambda y+\mu z)^{2}+q(y, z),
$$

where $q$ is an indefinite binary quadratic form. Let $e$ denote $m_{-}(q)$, so that for arbitrarily small $\rho \geqq 0$ we may write

$$
q(y, z) \sim q_{\rho}(y, z)=\frac{-e}{1-\rho}\left(y+\delta_{\rho} z\right)^{2}+\frac{d(1-\rho)}{e} z^{2},
$$

where $\delta_{\rho}$ depends on $\rho$ and satisfies $\left|\delta_{\rho}\right| \leqq \frac{1}{2}$. Then for arbitrarily small $\rho \geqq 0$ there exists a form $f_{\rho}$ such that

$$
f \sim f_{\rho}=\left(x+\lambda_{\rho} y+\mu_{\rho} z\right)^{2}+q_{\rho}(y, z),
$$

where $\lambda_{\rho}$ and $\mu_{\rho}$ depend on $\rho$.

Consider the section

of $f_{\rho}$. Clearly

$$
t(x, y)=\left(x+\lambda_{p} y\right)^{2}-e y^{2} /(1-\rho)
$$

$$
m_{+}(t)=1, m_{-}(t) \geqq m_{-}(f) \geqq k .
$$

Hence we may apply theorem 1 , with $\delta=0$, to $t$ to conclude that either

(i) $t \sim x^{2}-k x y-k y^{2}$, or

(ii) $d(t) \geqq\left(k^{2}+6 k+1\right) / 4$. 
Now one of these possibilities must be true for arbitrarily small $\rho$. If the second possibility holds for arbitrarily small $\rho$, we have that

$$
e /(1-\rho) \geqq\left(k^{2}+6 k+1\right) / 4=K / 4
$$

for arb. small $\rho$ and so $e \geqq K / 4$. Now $q$ cannot take any value in the open interval $\left(0, \frac{3}{4}\right)$, else by choosing $x$ suitably we could obtain a value of $f$ contradicting $m_{+}(f)=1$. Hence as $m_{-}(q)=e, q$ can take no values in the open interval $\left(-e, \frac{3}{4}\right)$. Then by the result of Segré

i.e.

$$
\begin{aligned}
d(q) & \geqq 3 e / 4+\frac{1}{4} \max \left(\frac{9}{16}, e^{2}\right), \\
d & \geqq 3 K / 16+K^{2} / 64=d_{1} .
\end{aligned}
$$

We now consider the case that the first possibility above, namely $t \sim x^{2}-k x y-k y^{2}$, occurs for arbitrarily small $\rho$. This implies that

$$
d(t)=e /(1-\rho)=\left(k^{2}+4 k\right) / 4
$$

for arb. small $\rho$. Hence our "arb. small $\rho$ " must be $\rho=0$, and so

$$
t=\left(x+\lambda_{0} y\right)^{2}-\frac{1}{4}\left(k^{2}+4 k\right) y^{2} .
$$

As this is equivalent to $x^{2}-k x y-k y^{2}$, a form with integral coefficients, we must have $\lambda_{0} \equiv k / 2(\bmod 1)$.

Suppose that $q_{0}$ takes a value in the open interval

$$
I=\left(-\left(k^{2}+6 k+1\right) / 4,-\left(k^{2}+4 k\right) / 4\right),
$$

say at the point $(y, z)=(Y, Z)$. Then choosing $x$ such that $\left(x+\lambda_{0} Y+\mu_{0} Z\right)^{2}$ lies in the closed interval

$$
\left[\left(k^{2}+2 k+1\right) / 4,\left(k^{2}+4 k+4\right) / 4\right]
$$

would give a value of $f_{0}$ lying in the open interval $(-k, 1)$, which, as $f \sim f_{0}$, contradicts either $m_{+}(f)=1$ or $m_{-}(t) \geqq k$. Hence $q_{0}$ can take no values in the interval $I$.

Suppose for the moment that the integers $g_{2 i}$ of the chain $\left[g_{i}\right]$ associated with $q_{0}$ (as in lemma 1 ) are bounded above by $S^{*}$. Then by applying lemma 1 to the form

$$
Q_{0}(x, y)=q_{0}(x, y) / 2 \sqrt{d\left(q_{0}\right)},
$$

taking $c_{1}=\frac{1}{2} C\left(S^{*}\right)$ and $c_{2}$ arbitrarily small, we may conclude that one of the following holds:
(a) $2 \sqrt{d\left(q_{0}\right)} / m_{-}\left(q_{0}\right)=2 \sqrt{ } d / e \geqq 2$,
(b) $2 \sqrt{d\left(q_{0}\right)} / m_{-}\left(q_{0}\right)=2 \sqrt{ } d / e \geqq \sqrt{1+4 / S^{*}}\left(\frac{k^{2}+6 k+1}{k^{2}+4 k}-c_{2}\right)$, 
(c) There exist integers $r$ and $s$, both at most $S^{*}$, such that for all $i$,

$$
g_{2 i+1}=1, \quad g_{4 i}=r, \quad g_{4 i+2}=s .
$$

If however, $g_{2 i}>S^{*}$ for at least one $i$, then either (a) above holds if $g_{j} \geqq 2$ for at least one odd $j$, or $g_{j}=1$ for all odd $j$ and $Q_{0}$ takes a value $m_{1}$ where

$$
1 / m_{1} \geqq\left(S^{*}+1, \overline{1}\right)+(0, \overline{1}) .
$$

This latter implies that

and so

$$
2 \sqrt{ } d / m_{+}\left(q_{0}\right) \geqq S^{*}+\sqrt{ } 5,
$$

$$
\left.2 \sqrt{ } d \geqq m_{+}\left(q_{0}\right) ! S^{*}+\sqrt{ } 5\right)
$$

Thus if the possibility (c) above does not hold, either

(i) $2 \sqrt{ } d \geqq 2 e$, or

(ii) $2 \sqrt{ } d \geqq e \sqrt{1+4 / S^{*}}\left(\left(k^{2}+6 k+1\right) /\left(k^{2}+4 k\right)-c_{2}\right)$

for arbitrarily small $c_{2}$, or

(iii) $2 \sqrt{ } d \geqq m_{+}\left(q_{0}\right)\left(S^{*}+\sqrt{ } 5\right)$.

From these we conclude that either

(i') $d \geqq e^{2}$, or

(ii') $d \geqq K^{2}\left(1+4 / S^{*}\right) / 64$, or

(iii') $d \geqq 9\left(S^{*}+\sqrt{ } \mathbf{5}\right)^{2} / 64$.

We shall now show that in each of these cases $d \geqq d_{2}$. Clearly it is only necessary to show that $e^{2} \geqq d_{2}$. For $k=2$, numerical evaluation shows that $S^{*}=6$ and that

$$
e^{2}=9>\frac{5}{3} \cdot \frac{289}{64}=d_{2} \text {. }
$$

As $t(S)$ (and hence $S^{*}$ ) is increasing with $k$ it follows that $S^{*} \geqq 6$ for $k \geqq 2$, and so

$$
d_{2} \leqq \frac{5}{3} \cdot \frac{1}{64}\left(k^{2}+6 k+1\right)^{2} .
$$

Now for $k \geqq 3$ it is a simple matter to verify that

$$
\frac{5}{3} \cdot \frac{1}{64}\left(k^{2}+6 k+1\right)^{2}<\left(\left(k^{2}+4 k\right) / 4\right)^{2},
$$

and hence $e^{2}>d_{2}$ as required.

Thus, summarising, we have proved so far that if $f$ satisfies the conditions of theorem 2 then either $d \geqq \min \left(d_{1}, d_{2}\right)$ or $f$ is equivalent to the form

where

$$
f_{0}=\left(x+\lambda_{0} y+\mu_{0} z\right)^{2}+q_{0}(y, z) \text {, }
$$




$$
\begin{aligned}
\lambda_{0} & \equiv \frac{1}{2} k(\bmod 1), \\
m_{-}\left(q_{0}\right) & =e=\left(k^{2}+4 k\right) / 4, \\
q_{0} & =-e\left(y+\delta_{0} z\right)^{2}+d z^{2} / e,
\end{aligned}
$$

and the chain of integers $\left[g_{i}\right]$ associated with $q_{0}$ has the property that there exist integers $r$ and $s$, both at most $S^{*}$, such that $g_{2 i+1}=1, g_{4 i}=r$, and $g_{4 i+2}=s$ for all integers $i$. Clearly, to complete the proof of theorem 2 , we need only show that $f_{0}$, with the above properties, must be equivalent to $f(r, s, l ; x, y, z)$ for some $l<s$, and that $m_{-}(f)=k$.

If $q_{0}$ has the above properties then

$$
\begin{aligned}
q_{0}(y, z) & \sim-e\left\{y^{2}-\frac{s(r+2)}{r s+r+s} y z-\frac{r+2}{r s+r+s} z^{2}\right\} \\
& \sim-e\left\{y^{2}-\frac{r(s+2)}{r s+r+s} y z-\frac{s+2}{r s+r+s} z^{2}\right\} .
\end{aligned}
$$

Hence by passing to an equivalent form if necessary we may take $f_{0}$ to be of the form

$$
(x+\lambda y+\mu z)^{2}-e q(r, s ; y, z)
$$

where we may assume without loss of generality that $r \leqq s \leqq S^{*}$. The congruence

$$
\lambda \equiv \frac{1}{2} k(\bmod 1)
$$

may be deduced in the same way that $\lambda_{0} \equiv \frac{1}{2} k$ was deduced. Hence $f$ is equivalent to the form

$$
f^{*}=\left(x+\frac{1}{2} k y+\mu z\right)^{2}-\frac{1}{4}\left(k^{2}+4 k\right) q(r, s ; y, z)
$$

which takes the value $-k$ at $(x, y, z)=(0,1,0)$. Thus as $m_{-}(f) \geqq k$ is given we must have $m_{-}(f)=k$. It now remains, to complete the proof of the theorem, to show that $\mu \equiv l / s(\bmod 1)$.

We have

$$
f^{*}(x, 1,-s)=\left(x+\frac{1}{2} k-\mu s\right)^{2}-\left(k^{2}+4 k\right) / 4,
$$

and so by choosing $x$ such that

$$
\frac{1}{2}(k+1) \leqq\left|x+\frac{1}{2} k-\mu s\right| \leqq \frac{1}{2}(k+2)
$$

we obtain a value of $f^{*}$ contradicting either $m_{+}(f)=1$ or $m_{-}(f) \geqq k$ unless

$$
\frac{1}{2} k-\mu s \equiv \frac{1}{2} k(\bmod 1) \text {. }
$$

That is, $\mu \equiv l / s$ for some $l$ with $0 \leqq l<s$. Hence

$$
f \sim f^{*} \sim\left(x+\frac{1}{2} k y+l z / s\right)^{2}-\frac{1}{4}\left(k^{2}+4 k\right) q(r, s ; y, z)
$$

as required. This completes the proof of theorem 2 . 
Further information about the relationship between $r, s$ and $l$ for those forms $f(r, s, l ; x, y, z)$ which do in fact have $m_{-}(f)=k$ and $m_{+}(f)=1$ may be obtained by applying various automorphs of $q(r, s ; y, z)$ and by applying various $x-y$ transformations. The following theorem gives some of these relationships.

THEOREM 3. Let $k \geqq 2$ be integral and let $d_{1}, d_{2}, S^{*}$ and $f=$ $f(r, s, l ; x, y, z)$ be defined as in theorem 2 . Let

$$
\begin{aligned}
B & =s(r+2) /(r s+r+s), \\
e & =\left(k^{2}+4 k\right) / 4, \\
E & =B\left(1+\frac{1}{2} k\right)+2 l / s, \quad \text { and } \\
F & =(l / s)^{2}-B\left(1-l\left(1+\frac{1}{2} k\right)\right) / s+e B^{2} / 4 .
\end{aligned}
$$

Then if $d(f)<\min \left(d_{1}, d_{2}\right)$ and if $m_{+}(f)=1$ and $m_{-}(f)=k$ the following conditions must be satisfied:

(i) $r(k / 2+l / s) \equiv 0(\bmod 1)$,

(ii) The fraction $\mathrm{seB}$, when reduced to its lowest form, has denominator at most $S^{*}$,

(iii) There exist positive integers $r^{\prime}$ and $s^{\prime}$, both at most $S^{*}$, and an integer $b$ such that

and

$$
E=2 b \pm B^{\prime}
$$

$$
-F=-b^{2} \pm b B^{\prime}+B^{\prime} / s^{\prime},
$$

where $B^{\prime}$ denotes the fraction

$$
s^{\prime}\left(r^{\prime}+2\right) /\left(r^{\prime} s^{\prime}+r^{\prime}+s^{\prime}\right),
$$

and (iv) For this $r^{\prime}$ and $s^{\prime}$,

$$
B^{2} / 4+B / s=\left(B^{\prime}\right)^{2} / 4+B^{\prime} / s^{\prime} .
$$

Proof. (i) Considering the section

$$
f(x, r+1, r)=\left(x+\frac{1}{2} k(r+1)+r l / s\right)^{2}-\left(k^{2}+4 k\right) / 4
$$

in the same way that the section $f^{*}(x, 1,-s)$ was considered in the proof of theorem 2 yields that

$$
\frac{1}{2} k(r+1)+r l / s \equiv \frac{1}{2} k(\bmod 1) .
$$

This clearly implies that

$$
r(k / 2+l / s) \equiv 0(\bmod 1) .
$$


(ii) Applying the transformation

$$
(x, y, z) \rightarrow(X, X-Y, Z)
$$

to $f$ yields the equivalent form

$$
\left(X+\frac{1}{2} k Y+D Z\right)^{2}-e\left(Y^{2}+E Y Z+F Z^{2}\right),
$$

where

$$
2 D=l(k+2) / s+e B .
$$

Repeating the argument of theorem 2 we find that

$$
Y^{2}+E Y Z+F Z^{2} \sim q\left(r^{\prime}, s^{\prime} ; \bar{y}, \bar{z}\right)
$$

for some $r^{\prime}$ and $s^{\prime}$ satisfying $r^{\prime} \leqq s^{\prime} \leqq S^{*}$. We now proceed to find out further information about the transformations yielding this equivalence.

Let $h$ be an integer such that

$$
0 \leqq|E+2 h| \leqq 1,
$$

and consider the transformation

$$
\begin{aligned}
& Y \rightarrow \bar{y}+h \bar{z} \\
& Z \rightarrow \bar{z} .
\end{aligned}
$$

This sends the form $Y^{2}+E Y Z+F Z^{2}$ into the form

$$
\begin{aligned}
q_{1}(\bar{y}, \bar{z}) & =\bar{y}^{2}+(E+2 h) \overline{y z}+\left(h^{2}+E h+F\right) \bar{z}^{2} \\
& =\bar{y}^{2}-E_{1} \overline{y z}-F_{1} \bar{z}^{2} .
\end{aligned}
$$

By changing the sign of $\bar{y}$ if necessary we may assume $E_{1}$ to be non-negative. Let $d$ denote $d(q)=d\left(q_{1}\right)$. Then as $s \geqq r$ we have $B \geqq 1$, and so

$$
E_{1}^{2}+4 F_{1}=4 d=B^{2}+4 B / s>1 \text {. }
$$

Hence as $E_{1}^{2} \leqq 1$ we find that $F_{1}>0$. We shall now show that $q_{1}(\bar{y}, \bar{z})$ is either $q\left(r^{\prime}, s^{\prime} ; \bar{y}, \bar{z}\right)$ or $q\left(s^{\prime}, r^{\prime} ; \bar{y}, \bar{z}\right)$.

Suppose that

$$
F_{1} \geqq \sqrt{ } d .
$$

Then $d=E_{1}^{2} / 4+F_{1} \geqq \sqrt{ } d$, and so $d \geqq 1$. However this leads to a contradiction as follows:

(a) If $s \geqq 2$, then

$$
d=1-\frac{3 r^{2} s^{2}-4 r s+4 r s^{2}-8 s+4 s r^{2}-8 r}{r(r s+r+s)^{2}}<1
$$

which contradicts $d \geqq 1$. 
(b) If $r=s=1$, the only other possibility, $d=\frac{5}{4}$ and $E, F$ and thus $F_{1}$ are integral. However $\frac{5}{4}=d \geqq F_{1} \geqq \sqrt{ } \frac{5}{4}$, and this is clearly insoluble in integers $F_{1}$.

From the above considerations it follows that $F_{1}<\sqrt{ } d$, and so, from a theorem of Lagrange, $-F_{1}$ occurs as a coefficient in one of the reduced forms equivalent to $q_{1}$ (and hence $q\left(r^{\prime}, s^{\prime} ; \bar{y}, \bar{z}\right)$ ). From the nature of the chain of integers $\left[g_{l}\right]$ associated with $q_{1}$ it follows that either

or

$$
F_{1}=\left(r^{\prime}+2\right) /\left(r^{\prime} s^{\prime}+r^{\prime}+s^{\prime}\right)
$$

$$
F_{1}=\left(s^{\prime}+2\right) /\left(r^{\prime} s^{\prime}+r^{\prime}+s^{\prime}\right) \text {. }
$$

Upon calculating $E_{1}$ from $d$ in terms of $r^{\prime}$ and $s^{\prime}$, it immediately becomes clear that $q_{1}$ is either $q\left(r^{\prime}, s^{\prime} ; \bar{y}, \bar{z}\right)$ or $q\left(s^{\prime}, r^{\prime} ; \bar{y}, \bar{z}\right)$. By dropping the assumption that $r^{\prime} \leqq s^{\prime}$ we may assume that

$$
q_{1}=q\left(r^{\prime}, s^{\prime} ; \bar{y}, \bar{z}\right) \text {. }
$$

Applying the transformation (5) to the form (3) yields the equivalent form

$$
\left(X+\frac{1}{2} k \bar{y}+\left(D+\frac{1}{2} k h\right) \bar{z}\right)^{2}-e q\left(r^{\prime}, s^{\prime} ; \bar{y}, \bar{z}\right) .
$$

Considering this form as in theorem 2 yields that

Hence

$$
D+\frac{1}{2} k h \equiv l^{\prime} / s^{\prime}(\bmod 1) \text {. }
$$

and so

$$
2 D=l(k+2) / s+e B \equiv 2 l^{\prime} / s^{\prime}(\bmod 1)
$$

$$
2 s D \equiv e B s \equiv 2 s l^{\prime} / s^{\prime}(\bmod 1) .
$$

Thus the denominator of the reduced form of the fraction $s e B$ divides $s^{\prime}$ and hence is at most $S^{*}$.

(iii) As $q_{1}=q\left(r^{\prime}, s^{\prime} ; \bar{y}, \bar{z}\right)$ it follows upon sorting out the relations between $h, E, F, E_{1}$ and $F_{1}$ that

and

$$
B^{\prime}=E_{1}= \pm(E+2 h)
$$

$$
B^{\prime} / s^{\prime}=F_{1}=-\left(h^{2}+E h+F\right) .
$$

The required integer $b$ is then given by $b=-h$.

(iv) Equating the determinants of $q$ and $q_{1}$ yields that

$$
B^{2} / 4+B / s=\left(B^{\prime}\right)^{2} / 4+B^{\prime} / s^{\prime} .
$$

It should be noticed that condition (iv) of theorem 3 is highly restrictive. Calculations performed on the C.S.I.R.O.'s C.D.C. " 3200 " computer in Adelaide have shown that for $S^{*} \leqq 200$, the couple $\left(r^{\prime}, s^{\prime}\right)$ must be either $(r, s)$ or $(s, r)$. 


\section{Acknowledgement}

These results were obtained while I held a C.S.I.R.O. Senior Postgraduate Studentship.

\section{References}

[1] L. E. Dickson, Introduction to the theory of numbers (University of Chicago Press, 1929). [2] A. Oppenheim, 'Value of quadratic forms', Quart. J. Math. Oxford (2) 4 (1953), 54-59.

[3] M. B. Segré, 'Lattice Points in infinite domains and asymmetric diophantine approximations, Duke Math. J. 12 (1945), 337-365.

[4] L. Tornheim, 'Asymmetric minima of quadratic forms and asymmetric diophantine approximation, Duke Math. J. 22 (1955), 287-294.

The University of Adelaide

Adelaide, S.A. 